\title{
Treatment of Venous Thromboembolism in Cancer Patients: A Systemic Review and Meta-analysis on the Efficacy and Safety of Different DOACs
}

\section{Xiaojun Song}

Peking Union Medical College Hospital

Zhili Liu

Peking Union Medical College Hospital Eastern Branch: Peking Union Medical College Hospital

Rong Zeng

Peking Union Medical College Hospital

Jiang Shao

Peking Union Medical College Hospital

\section{Bao Liu}

Peking Union Medical College Hospital

\section{Yuehong Zheng}

Peking Union Medical College Hospital

Changwei Liu

Peking Union Medical College Hospital

Wei Ye ( $\nabla$ xiepan963114@163.com )

Peking Union Medical College Hospital

\section{Research}

Keywords: direct oral anticoagulant, low molecular weight heparin, venous thromboembolism, cancer, major bleeding evets

Posted Date: November 4th, 2020

DOI: https://doi.org/10.21203/rs.3.rs-100576/v1

License: (c) (i) This work is licensed under a Creative Commons Attribution 4.0 International License. Read Full License 


\section{Abstract}

Background: To evaluate the efficacy and safety of different direct oral anticoagulants (DOACs) compared with low molecular weight heparin (LMWHs) in the treatment of venous thromboembolism (VTE) in cancer patients.

Methods: Literature was searched in databases including Cochrane Library, EMBASE (Ovid) and MEDLINE (Pubmed). Eligible studies were included and data were collected independently by two reviewers. We conducted a systemic review of the efficacy and safety of DOACs in the treatment of VTE in cancer patients. The odds ratios of different DOACs compared with LMWHs for VTE, DVT, PE recurrences, major bleeding and clinically relevant non-major bleeding (CRNMB) were calculated in metaanalyses and subgroup analyses.

Results: A total of 18 articles were eligible for analyses, including 4 randomized controlled trials (RCTs) and 14 retrospective studies. Both RCTs and retrospective studies confirmed that DOACs decreased the risk of VTE recurrence (RCTs: OR, $0.60 ; 95 \% \mathrm{Cl}, 0.45-0.80$; retrospective studies: OR, $0.73 ; 95 \% \mathrm{Cl}, 0.59-0.90$ ) and DVT recurrence (RCTs: OR, 0.54; $95 \% \mathrm{Cl}, 0.36-0.80$; retrospective studies: $\mathrm{OR}, 0.20 ; 95 \% \mathrm{Cl}, 0.06-0.63$ ), but not $\mathrm{PE}$ recurrence or fatal $\mathrm{PE}$ in cancer patients. Subgroup analyses revealed an important role of rivaroxaban in decreasing recurrent VTE. Meanwhile, major bleeding events were not increased in the DOAC group, but the risk of CRNMBs were significantly elevated. Subgroup analyses confirmed the role of rivaroxaban in increasing the risk of major bleeding events and CRNMBs.

Conclusions: Compared with LMWHs, DOACs, especially rivaroxaban, significantly reduced the risk of VTE and DVT, rather than PE, recurrence in patients with cancer. Although DOACs did not increase the major bleeding events in pooled analysis, rivaroxaban was showed an elevated risk of this adverse effect in subgroup analysis. In addition, the risk of CRNMB events was increased after the application of DOACs including rivaroxaban.

\section{Background}

Venous thromboembolism (VTE) in cancer patients is associated with increased mortality (1). It was estimated that the incidence of recurrent VTE was about 9.6\%, and in particular, the incidence was as high as $22.1 \%$ within the six months from cancer diagnosis(2). The common forms of VTE include deep vein thrombosis (DVT) and pulmonary embolism (PE)(3). Given the detrimental effect of VTE on cancer patients, anticoagulant therapy is recommended for the treatment of VTE in patients was active cancer(4). However, anticoagulant treatment leads to the risk of bleeding(5), which is the most severe adverse effect in clinical practice. Therefore, in cancer patients, it should carefully consider the balance between anticoagulation and bleeding prevention in the management of VTE.

Low molecular weight heparin (LMWHs) is the traditionally recommended therapy for the VTE treatment, which significantly decreased the recurrence of VTE(6). Nevertheless, direct oral anticoagulants (DOACs), which include rivaroxaban, edoxaban, apixaban and others, are more and more recognized as promising 
strategy for decreasing VTE events in cancer patients, as many recent studies showed that DOACs are comparable with LMWHs in the efficacy of VTE treatment in cancer patients(7-9). Currently, there is lack of systemic review and meta-analysis investigating effects of different DOACs on the treatment of VTE, as well as adverse effects, in patients with cancer.

In order to clarify the efficacy and safety of different DOACs for the treatment of recurrent VTE in cancer patients, we systemically reviewed and performed meta-analysis with subgroup analyses of the observational studies and randomized controlled trials concerning the DOACs compared with LMWHs in treating VTE. We hope to provide updated evidences for clinical decision-making on anticoagulant therapy in cancer patients.

\section{Methods}

\section{Search strategy}

This systemic review and meta-analysis were conducted according to the Preferred Reporting Items for Systematic Reviews and Meta-Analyses (PRISMA)(10). The literature was systematically searched in the database including Cochrane Library, EMBASE (Ovid) and MEDLINE (PubMed) with a publication date from initiation to October 1, 2020. We used the keywords "oral anticoagulant" or "Factor Xa inhibitor" or "rivaroxaban" or "edoxaban" or "apixaban" or "dabigatran", "low molecular weight heparin", "cancer" or "tumor" or "neoplasm", "venous thromboembolism" or "vein thrombosis" to search all the potentially relevant literature.

\section{Study collection and data extraction}

Two investigators independently analyzed the relevant literature for further study collection and data extraction. When an investigator was uncertain for inclusion or exclusion of an article, or there was discrepancy between the two reviewers about an article, it was determined by in-depth discussion. The relevant literature was first screened by tittle, and then identified with further reading of the abstract. An article was finally included if it was met the inclusion criteria after reviewing of the full text. Inclusion criteria: a. retrospective observational studies or prospective randomized controlled trials; b. studies investigating the efficacy and safety of oral anticoagulant and low molecular weight heparin for the treatment of venous thromboembolism in cancer patients; c. the follow-up for anticoagulation therapy was not less than 6 months; Exclusion criteria: a. studies using DOAC and LMWH for prophylaxis of venous thromboembolism; $b$. studies with incomplete data that impeding the systemic review and metaanalysis; c. reviews, case reports, abstracts for academic conference and other research types that were not appropriate for systemic review and meta-analysis. For the eligible literature, study type, period, grouping, sample size, treatment dose, treatment duration, follow-up, recurrent VTE, recurrent PE, major bleeding, and clinically relevant non-major bleeding (CRNMB) events were extracted.

\section{Main outcomes}


The main outcomes for evaluating efficacy includes recurrence of venous thromboembolism (VTE), deep venous thrombosis or pulmonary embolism within 6 months, as well as fatal pulmonary embolism. The outcomes for safety include major bleeding and clinically relevant non-major bleeding (CRNMB) events.

\section{Quality assessment}

In order to assess the risk of bias, we used the Risk of Bias in Non-randomized Studies-of Interventions (ROBINS-I) for evaluating the observational cohort studies. ROBINS-I includes assessing bias due to confounding, in selection of participants into the study, in classification of intervention, due to deviations from intended interventions, due to missing data, in measurement of outcomes, and in selection of the reported result. Revised Cochrane Risk of Bias Tool (RoB2.0) was used for evaluating bias of the RCTs. Publication bias was visually assessed by the symmetry of results on the funnel plots.

\section{Statistical analyses}

Stata 12.0 was used for statistical meta-analyses. The pooled odds ratios (ORs) and corresponding 95\% confidence interval were calculated for the outcomes evaluating efficacy and safety of DOACs and LMWH in the treatment of venous thromboembolism in cancer patients, as well as in subgroup analyses. The Cochran $Q$ test and $P$ statistic test were used for evaluating the heterogeneity of included studies. A $P$ value $<0.01$ in Q test or $P^{2}$ value $>50 \%$ represented significant heterogeneity among the studies, and random effects model was used for meta-analysis, otherwise the fixed effects model was used for pooled analysis.

\section{Results}

\section{Study characteristics}

A total of 1023 articles were obtained after the initial retrieval with the relevant keywords. The flow-chart for identifying articles for final inclusion was illustrated in Fig. 1. After screening for tittle and abstract, 43 articles were identified for the comprehensive review of full text. Among these literature, 16 articles were reviews; 4 articles were absence of valid data; 3 articles reported the same population or from the same institution; 2 articles were lack of control group. Eventually, 18 articles were identified for final analyses, including 4 RCTs and 14 retrospective cohort studies, with a total of 7319 patients with cancer. Among these patients, 3430 cases $(46.9 \%)$ and 3889 cases (53.1\%) received DOAC and LMWH treatment, respectively. The characteristics results of quality assessment of included studies were shown in Supplementary Tables 1 and 2, respectively.

In terms of DOACs, 10 studies (including 1 RCT) compared the efficacy and safety of rivaroxaban and LMWH in the treatment of venous thromboembolism(11-19); apixaban was used in 3 studies (including 2 RCTs)(19-21); edoxaban was given in 1 RCT(22); 5 studies did not specify the oral anticoagulant used in the trials(23-27). The duration of follow-up was from 6 to 24 months in these studies. 


\section{Efficacy of DOACs compared with LWMH in the treatment of VTE}

First, we investigated the pooled odds ratio of DOACs compared with LWMH for treatment of VTE in cancer patients. There is no significant heterogeneity of the included studies $(P=0 \%, P=0.921$, Fig. 2$)$, so the fixed effects model was used. The results of pooled analysis showed that DOACs significantly reduced the VTE recurrence in patients with cancer (OR: $0.68,95 \% \mathrm{Cl}: 0.57-0.8, P<0.001$, Fig. 2). Funnel plot did not show significant publication bias of these studies (Supplementary Fig. 1). The ORs for RCTs and retrospective studies were further calculated respectively. Similarly, both RCTs and retrospective cohort studies confirmed a reduced risk of VTE recurrence in cancer patients (RCTs: OR, $0.60 ; 95 \% \mathrm{Cl}$, $0.45-0.80 ; P<0.001$; heterogeneity, $P=24.9 \% ; P=0.262$; retrospective studies: $\mathrm{OR}, 0.73 ; 95 \% \mathrm{Cl}, 0.59-$ $0.90 ; P=0.003$; heterogeneity, $P^{2}=0 \% ; P=0.979$, Fig. 2).

In order to clarify the efficacy of different DAOCs for VTE treatment, we further investigated the ORs for VTE recurrence of edoxaban, rivaroxaban, and apixaban, respectively. Interestingly, results showed that only rivaroxaban was correlated with lower risk of VTE recurrence in cancer patients $(\mathrm{OR}, 0.69 ; 95 \% \mathrm{Cl}$, $0.54-0.87 ; P=0.002$; heterogeneity, $P^{2}=0.0 \% ; P=0.893$, Fig. 3 ). These results confirmed that DOAC, especially rivaroxaban, treatment can significantly decrease the VTE recurrence risk in patients with cancer.

Second, the risk of DVT recurrence was compared between DOACs and LWMHs. A total of 9 studies evaluated the DVT recurrence risk in patients using DOACs or LWMHs. Pooled analysis showed a significant decreased DVT recurrence in DOAC group compared with that of LWMH group (OR: 0.47, 95\%Cl: $0.32-0.67, P<0.001$, heterogeneity, $P^{2}=23.4 \% ; P=0.236$, Fig. 4$)$. We did not find significant publication bias of the included studies on the funnel plot (Supplementary Fig. 2). Consistently, RCTs and retrospective studies obtained similar results for DOACs (RCTs: OR, 0.54; 95\%Cl, 0.36-0.80; $P=0.002$; heterogeneity, $P^{2}=16.5 \% ; P=0.309$; retrospective studies: $O R, 0.20 ; 95 \% \mathrm{Cl}, 0.06-0.63 ; P=0.006$; heterogeneity, $P^{2}=34 \% ; P=0.194$, Fig. 4).

Subgroup analysis revealed that only rivaroxaban was effective in reducing the DVT recurrence risk compared with LWMHs (OR: 0.31, 95\%Cl: $0.14-0.66, P<0.001$, heterogeneity, $P=1.5 \% ; P=0.362$, Fig. 5). Only one study investigated the effect of edoxaban and LWMHs on DVT recurrence risk, and it was found that edoxaban reduced the risk of DVT recurrence in cancer patients (OR: $0.553,95 \% \mathrm{Cl}: 0.30-0.94$, Fig. 5). Other two studies did not find significant relation between apixaban medication and reduced risk of DVT recurrence (OR: $0.62,95 \% \mathrm{Cl}: 0.31-1.24$, heterogeneity, $P^{2}=65.5 \% ; P=0.089$, Fig. 5). Thus, these resulted confirmed that DOACs, especially rivaroxaban, can reduce the DVT recurrence risk in cancer patients (Supplementary Fig. 3).

Third, we conducted a meta-analysis on the effect of DOACs on PE recurrence. Results showed that the pooled OR and $95 \% \mathrm{CI}$ for DOACs was $0.75(0.53,1.05)$, indicating that DOACs did not reduce the risk of 
PE recurrence compared with that of LMWHs (Heterogeneity, $P^{2}=0.0 \% ; P=0.664$, Fig. 6). Consistent with the pooled analysis, subgroup analyses did not find significant difference in PE recurrence risk between LMWHs and edoxaban, rivaroxaban, and apixaban, respectively (Supplementary Fig. 4). In addition, there was no significant difference in fatal PE recurrence between DOACs and LMWHs in cancer patients (Supplementary Fig. 5).

\section{Safety of DOACs compared with LWMHs}

In order to evaluate the safety of DOACs compared with LWMHs, we investigated the occurrence of major bleeding events and CRNMB events in the treatment of VTE. Pooled analysis showed that DOACs did not increase the risk of major bleeding events in cancer patients (OR: $1.04,95 \% \mathrm{Cl}: 0.84-1.28$, heterogeneity, $P^{2}=0.0 \% ; P=0.490$, Fig. 7). However, subgroup analysis revealed an increased risk of major bleeding events in rivaroxaban group compared with LMWH group (OR: $1.36,95 \% \mathrm{Cl}: 1.01-1.82$, heterogeneity, $P^{2}=$ $0.0 \% ; P=0.714$, Fig. 8). (Supplementary Fig. 6).

Finally, we found that DOACs significantly increased the risk of CRNMB in cancer patients (OR: 1.45, 95\%Cl: $1.21-1.73$, heterogeneity, $\mathrm{I}^{2}=48.3 \%$; $\mathrm{P}=0.022$, Fig. 9), which was similar in RCTs (OR: $1.58,95 \% \mathrm{Cl}$ : 1.22-2.04, heterogeneity, $P^{2}=42.3 \% ; P=0.158$, Fig. 9) and retrospective studies (OR: $1.33,95 \% \mathrm{Cl}$ : $1.03-$ 1.70 , heterogeneity, $P^{2}=53.6 \% ; P=0.022$, Fig. 9). (Supplementary Fig. 7).Subgroup analysis confirmed that the increase of CRNMB events was only observed in rivaroxaban group compared with LMWH group (OR: $2.41,95 \% \mathrm{Cl}$ : $1.75-3.31$, heterogeneity, $P^{2}=0.0 \% ; P=0.467$, Fig. 10 ).

\section{Discussion}

In this study, the pooled results of meta-analysis showed that DOACs significantly reduced the occurrence of recurrent VTE and DVT, but not PE or fatal PE, compared with LMWHs in patients with cancer. Subgroup analyses revealed an important role of rivaroxaban in decreasing the VTE and DVT recurrence. In addition, DOACs did not increase the risk of major bleeding events, but the risk of CRNMBs were increased. Subgroup analyses confirmed the role of rivaroxaban in increasing the risk of major bleeding events and CRNMBs.

Anticoagulant therapy is a complex clinical issue in cancer patients because it should balance between anticoagulation and bleeding. Traditionally, LMWHs are the preferred drugs for anticoagulant therapy in the prophylaxis and treatment of VTE, which were recommended by many guidelines(4). It has been well established that LMWHs was effective in reducing the VTE and overall mortality(28), though the risk of bleeding was still in debate(4). In recent years, the role of DOACs in the treatment of cancer-associated VTE has been increasingly recognized. A series of reviews and meta-analyses showed that DOACs was better than the LMWHs in preventing the recurrent VTE in cancer patients $(29,30)$. Similarly, DOACs were effective in reducing the DVT recurrence $(9,31)$, but not PE recurrence(31). In consistent with these results, we confirmed in our meta-analysis that the risk of VTE recurrence, as well as DVT recurrence, was 
significantly lower in patients treated with DOACs than that of LMWHs. But there was no significant difference in the risk of recurrent PE or fatal PE between the two groups.

Since DOACs include rivaroxaban, edoxaban, apixaban, etc., we further analyzed the risk effects of different kinds of DOACs in cancer patients by subgroup analyses. One RCT compared the efficacy of edoxaban with $\mathrm{LMWH}(22)$, but no significant difference in VTE recurrence was detected. Ten studies(1, 9, $12-16,18,19)$ including one RCT $(11)$ investigated the risk of recurrent VTE in patients receiving rivaroxaban compared with that of LMWH. The occurrence of VTE recurrence was significantly lower in rivaroxaban group, suggesting a role of rivaroxaban in reducing the recurrent VTE compared with that of LMWH. After excluding the RCT in meta-analysis, the difference in VTE recurrence between the two groups was still significant, further confirming the association between lower VTE risk and rivaroxaban treatment. Two RCTs $(20,21)$ and one retrospective study (19) compared the effect of apixaban with LMWH, and the results did not reveal significant OR for apixaban in VTE risk. However, when we excluded the retrospective study, pooled analysis of the two RCTs did demonstrate lower VTE risk in patients with apixaban. So, more RCTs are needed to further confirm the role of apixaban in preventing the VTE recurrence in cancer patients.

DVT events and PE events are the main manifestations of VTE in patients with cancer. Our study found that DOACs was superior than LMWHs in preventing recurrent DVT, which was consistent with previous studies $(9,31)$. Subgroup analyses were performed in edoxaban, rivaroxaban and apixaban groups based on the available data. Only one RCT(22) investigated the DVT recurrence between edoxaban group and LMWH group and results showed a lower risk of recurrent DVT in patients receiving edoxaban. One RCT(11) and two retrospective studies $(12,14)$ compared the efficacy of rivaroxaban and LMWHs, and results demonstrated that riaroxaban significantly reduced the DVT recurrence in cancer patients. Two $\operatorname{RCTs}(20,21)$ on apixaban found that it had no effect on reducing the DVT recurrence compared with that of LMWHs. Moreover, pooled analysis, as well as subgroup analyses, of the included studied did not show a superior effect of DOACs, including edoxaban, rivaroxaban and apixaban, upon LMWHs to reduce the risk of recurrent PE and fatal PE. According to the limited data, these results indicated that rivaroxaban was particularly important in reducing the risk of VTE recurrence in cancer patients. Further studies are still needed to clarify the role of other DOACs in the treatment of patients with cancer.

Currently, there is inconsistency about the effect of DOACs on major bleeding events in cancer patients. Some studies revealed that DOACs would increase the risk of major bleeding(9) but other studies did not find such an elevated risk(31). In our pooled analysis, regardless of RCTs or retrospective studies, there was no significant difference in the occurrence of major bleeding events between DOACs and LMWHs. However, subgroup analyses showed that rivaroxaban did increase the major bleeding events in cancer patients while edoxaban and apixaban did not induce elevated risk of major bleeding events. Thus, it is important for clinicians to consider the characteristics of different DOACs in patients with cancer. Consistent with previous studies $(9,31)$, our analysis confirmed that DOACs increased the risk of CRNMBs, suggesting a tendency of promoting bleeding of DOACs. However, one RCT about edoxaban found that the CRNMBs risk was not increased after treatment(22). Two RCTs(20, 21) and one 
retrospective study(19) consistently showed that apixaban did not increase the risk of CRNMB events in patients with cancer. Therefore, edoxaban and apixaban may be safer than the rivaroxaban in the treatment of VTE in cancer patients.

This study has some limitations. First, due to the limited data on different kinds of DOACs, the studies comparing the edoxaban, apixaban and LMWHs are rare. So, the conclusions in this study still needed be further confirmed. Second, the number of RCTs for different DOACs are somewhat small, which is only one or two RCTs in meta-analysis. Multi-center RCTs with large sample size are needed to further investigate the efficacy and safety of specific DOAC. Third, the subgroups of different kinds of cancers were not analyzed, which may influence the efficacy and safety of DOACs(31). Future studies should focus on the effects of different DOACs on specific cancers so as to help clinicians better determine the treatment strategy for VTE in patients with different cancers.

\section{Abbreviations}

DOACs: different direct oral anticoagulants

LMWHs: low molecular weight heparin

VTE: venous thromboembolism

PE: pulmonary embolism

DVT: deep vein thrombosis

\section{Declarations}

\section{Ethics approval and consent to participate}

Not applicable

\section{Consent for publication}

Not applicable

\section{Availability of data and material}

The dataset supporting the conclusions of this article is included within the article.

\section{Competing interests}

The authors declare that they have no competing interests.

\section{Funding}


None

\section{Authors' contributions}

XS and WY conceived and designed this meta-analysis. ZL, RZ and JS searched literatures. BL, YZ and $\mathrm{CL}$ analyzed data. XS and WY wrote the manuscript. All authors have approved the final manuscript as submitted.

\section{Acknowledgments}

None

\section{References}

1. Timp JF, Braekkan SK, Versteeg HH, Cannegieter SC. Epidemiology of cancer-associated venous thrombosis. Blood. 2013;122(10):1712-23.

2. Cohen AT, Katholing A, Rietbrock S, Bamber L, Martinez C. Epidemiology of first and recurrent venous thromboembolism in patients with active cancer. A population-based cohort study. Thromb Haemost. 2017;117(1):57-65.

3. Geerts WH, Bergqvist D, Pineo GF, Heit JA, Samama CM, Lassen MR, et al. Prevention of venous thromboembolism: American College of Chest Physicians Evidence-Based Clinical Practice Guidelines (8th Edition). Chest. 2008;133(6 Suppl):381S-453S.

4. Farge D, Bounameaux H, Brenner B, Cajfinger F, Debourdeau P, Khorana AA, et al. International clinical practice guidelines including guidance for direct oral anticoagulants in the treatment and prophylaxis of venous thromboembolism in patients with cancer. Lancet Oncol. 2016;17(10):e452-e66.

5. Kamphuisen PW, Beyer-Westendorf J. Bleeding complications during anticoagulant treatment in patients with cancer. Thromb Res. 2014;133 Suppl 2:S49-55.

6. Kearon C, Akl EA, Ornelas J, Blaivas A, Jimenez D, Bounameaux H, et al. Antithrombotic Therapy for VTE Disease: CHEST Guideline and Expert Panel Report. Chest. 2016;149(2):315-52.

7. Song AB, Rosovsky RP, Connors JM, Al-Samkari H. Direct oral anticoagulants for treatment and prevention of venous thromboembolism in cancer patients. Vasc Health Risk Manag. 2019;15:17586.

8. Franco-Moreno A, Cabezon-Gutierrez L, Palka-Kotlowsa M, Villamayor-Delgado M, Garcia-Navarro M. Evaluation of direct oral anticoagulants for the treatment of cancer-associated thrombosis: an update. J Thromb Thrombolysis. 2019;47(3):409-19.

9. Li A, Garcia DA, Lyman GH, Carrier M. Direct oral anticoagulant (DOAC) versus low-molecular-weight heparin (LMWH) for treatment of cancer associated thrombosis (CAT): A systematic review and meta-analysis. Thromb Res. 2019;173:158-63.

10. Moher D, Shamseer L, Clarke M, Ghersi D, Liberati A, Petticrew M, et al. Preferred reporting items for systematic review and meta-analysis protocols (PRISMA-P) 2015 statement. Syst Rev. 2015;4:1. 
11. Young AM, Marshall A, Thirlwall J, Chapman O, Lokare A, Hill C, et al. Comparison of an Oral Factor Xa Inhibitor With Low Molecular Weight Heparin in Patients With Cancer With Venous Thromboembolism: Results of a Randomized Trial (SELECT-D). J Clin Oncol. 2018;36(20):2017-23.

12. Chaudhury A, Balakrishnan A, Thai C, Holmstrom B, Nanjappa S, Ma Z, et al. The Efficacy and Safety of Rivaroxaban and Dalteparin in the Treatment of Cancer Associated Venous Thrombosis. Indian $\mathrm{J}$ Hematol Blood Transfus. 2018;34(3):530-4.

13. Signorelli JR, Gandhi AS. Evaluation of rivaroxaban use in patients with gynecologic malignancies at an academic medical center: A pilot study. J Oncol Pharm Pract. 2019;25(2):362-8.

14. Nicklaus MD, Ludwig SL, Kettle JK. Recurrence of malignancy-associated venous thromboembolism among patients treated with rivaroxaban compared to enoxaparin. J Oncol Pharm Pract. 2018;24(3):185-9.

15. Simmons B, Wysokinski W, Saadiq RA, Bott-Kitslaar D, Henkin S, Casanegra A, et al. Efficacy and safety of rivaroxaban compared to enoxaparin in treatment of cancer-associated venous thromboembolism. Eur J Haematol. 2018.

16. Streiff MB, Milentijevic D, McCrae K, Yannicelli D, Fortier J, Nelson WW, et al. Effectiveness and safety of anticoagulants for the treatment of venous thromboembolism in patients with cancer. Am J Hematol. 2018;93(5):664-71.

17. Lee JH, Oh YM, Lee SD, Lee JS. Rivaroxaban versus Low-Molecular-Weight Heparin for Venous Thromboembolism in Gastrointestinal and Pancreatobiliary Cancer. J Korean Med Sci. 2019;34(21):e160.

18. Lee JH, Hyun DG, Choi CM, Lee JC, Kim WS, Oh YM, et al. A Retrospective Study on Efficacy and Safety of Rivaroxaban and Dalteparin for Long-Term Treatment of Venous Thromboembolism in Patients with Lung Cancer. Respiration. 2019;98(3):203-11.

19. Wysokinski WE, Houghton DE, Casanegra Al, Vlazny DT, Bott-Kitslaar DM, Froehling DA, et al. Comparison of apixaban to rivaroxaban and enoxaparin in acute cancer-associated venous thromboembolism. Am J Hematol. 2019;94(11):1185-92.

20. McBane RD, 2nd, Wysokinski WE, Le-Rademacher JG, Zemla T, Ashrani A, Tafur A, et al. Apixaban and dalteparin in active malignancy-associated venous thromboembolism: The ADAM VTE trial. J Thromb Haemost. 2020;18(2):411-21.

21. Agnelli G, Becattini C, Meyer G, Munoz A, Huisman MV, Connors JM, et al. Apixaban for the Treatment of Venous Thromboembolism Associated with Cancer. N Engl J Med. 2020;382(17):1599-607.

22. Raskob GE, van Es N, Verhamme P, Carrier M, Di Nisio M, Garcia D, et al. Edoxaban for the Treatment of Cancer-Associated Venous Thromboembolism. N Engl J Med. 2018;378(7):615-24.

23. Alzghari SK, Seago SE, Garza JE, Hashimie YF, Baty KA, Evans MF, et al. Retrospective comparison of low molecular weight heparin vs. warfarin vs. oral Xa inhibitors for the prevention of recurrent venous thromboembolism in oncology patients: The Re-CLOT study. J Oncol Pharm Pract. 2018;24(7):494500 . 
24. Xiang E, Ahuja T, Raco V, Cirrone F, Green D, Papadopoulos J. Anticoagulation prescribing patterns in patients with cancer. J Thromb Thrombolysis. 2018;45(1):89-98.

25. Phelps MK, Wiczer TE, Erdeljac HP, Van Deusen KR, Porter K, Philips G, et al. A single center retrospective cohort study comparing low-molecular-weight heparins to direct oral anticoagulants for the treatment of venous thromboembolism in patients with cancer - A real world experience. $\mathrm{J}$ Oncol Pharm Pract. 2019;25(4):793-800.

26. Uppuluri EM, Burke KR, Haaf CM, Shapiro NL. Assessment of venous thromboembolism treatment in patients with cancer on low molecular weight heparin, warfarin, and the direct oral anticoagulants. $J$ Oncol Pharm Pract. 2019;25(2):261-8.

27. Pritchard ER, Murillo JR, Jr., Putney D, Hobaugh EC. Single-center, retrospective evaluation of safety and efficacy of direct oral anticoagulants versus low-molecular-weight heparin and vitamin $\mathrm{K}$ antagonist in patients with cancer. J Oncol Pharm Pract. 2019;25(1):52-9.

28. Akl EA, Kahale L, Neumann I, Barba M, Sperati F, Terrenato I, et al. Anticoagulation for the initial treatment of venous thromboembolism in patients with cancer. Cochrane Database Syst Rev. 2014(6):CD006649.

29. Rossel A, Robert-Ebadi H, Combescure C, Grosgurin O, Stirnemann J, Addeo A, et al. Anticoagulant therapy for acute venous thrombo-embolism in cancer patients: A systematic review and network meta-analysis. PLoS One. 2019;14(3):e0213940.

30. Sobieraj DM, Baker WL, Smith E, Sasiela K, Trexler SE, Kim O, et al. Anticoagulation for the Treatment of Cancer-Associated Thrombosis: A Systematic Review and Network Meta-Analysis of Randomized Trials. Clin Appl Thromb Hemost. 2018;24(9_suppl):182S-7S.

31. Haykal T, Zayed Y, Deliwala S, Kerbage J, Ponnapalli A, Malladi S, et al. Direct oral anticoagulant versus low-molecular-weight heparin for treatment of venous thromboembolism in cancer patients: An updated meta-analysis of randomized controlled trials. Thromb Res. 2020;194:57-65.

\section{Figures}




$\begin{aligned} & \text { Articles retrieved from } \\ & \text { database in initial searching } \\ & n=1023\end{aligned}$
\[ \]

\begin{tabular}{|l|l|}
\hline 142 articles identified & $\begin{array}{l}\text { Screened for tittle, 881 } \\
\text { articles excluded }\end{array}$ \\
\hline 43 articles identified & $\begin{array}{l}\text { Screened for abstract, 99 } \\
\text { articles excluded }\end{array}$ \\
\hline & $\begin{array}{l}\text { Screened for full text, } \\
\text { Reviews, } \mathrm{n}=16 ; \\
\text { Unavailable data, } \mathrm{n}=4 ; \\
\text { Same population or same research } \\
\text { institution, } \mathrm{n}=3 ; \\
\text { Did not meet the inclusion criteria } \\
\text { or lack of control group, n=2 }\end{array}$ \\
\hline 18 articles identified
\end{tabular}

Figure 1

Flow chart of screening the eligible articles 
Study

ID
$\%$

OR $(95 \% \mathrm{Cl})$

Weight

\section{RCT}

Raskob (2018)

Young (2018)

McBane II (2019)

Agnelli (2020)

Subtotal $(\mathrm{I}$-squared $=24.9 \%, p=0.262$ )

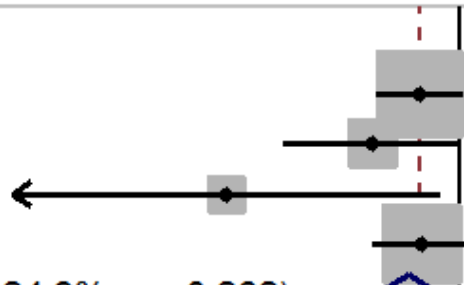

$0.67(0.44,1.02) \quad 16.74$

$0.42(0.18,0.99) \quad 5.33$

$0.10(0.01,0.82) \quad 2.79$

$0.68(0.43,1.09) \quad 13.37$

$0.60(0.45,0.80) \quad 38.23$

Retrospective study

Chaudhury (2018)

Ross (2016)

Signorelli (2019)

Alzghari (2018)

Streiff (2018)

Xiang (2018)

Simmons (2018)

Nicklaus (2018)

Phelps (2018)

Pritchard (2017)

Uppuluri (2017)

Lee (2019)

Wysokinski (2019)

Lee (2019)

Subtotal $(\mathrm{I}$-squared $=0.0 \%, p=0.979$ )

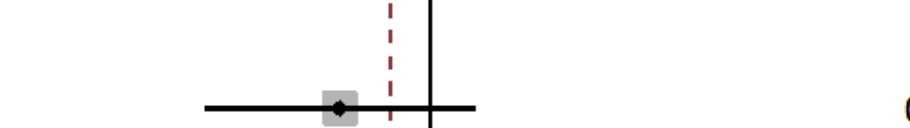

$0.41(0.11,1.54) \quad 2.46$

$0.57(0.07,4.83) \quad 0.82$

$0.46(0.02,11.92) \quad 0.37$

$0.44(0.08,2.40) \quad 1.17$

$0.71(0.53,0.95) \quad 32.23$

$0.45(0.13,1.54) \quad 2.51$

$0.65(0.30,1.42) \quad 5.10$

$0.63(0.17,2.42) \quad 1.69$

$0.86(0.41,1.80) \quad 4.80$

$0.62(0.22,1.76) \quad 2.87$

$0.98(0.11,8.63) \quad 0.51$

$0.98(0.25,3.77) \quad 1.32$

$0.99(0.50,1.96) \quad 5.16$

$2.00(0.41,9.91) \quad 0.75$

$0.73(0.59,0.90) \quad 61.77$

$0.68(0.57,0.80) \quad 100.00$

Overall $(I$-squared $=0.0 \%, p=0.921)$

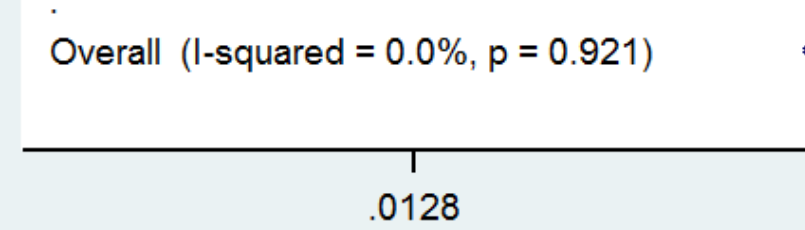




\section{Edoxaban}

Raskob (2018)

Subtotal $(1-$ squared $=. \%, p=$ )

$0.67(0.44,1.02)$

16.78

$0.67(0.44,1.02)$

16.78

Rivaroxaban

Young (2018)

Chaudhury (2018)

Ross (2016)

Signorelli (2019)

Streiff (2018)

Simmons (2018)

Nicklaus (2018)

Lee (2019)

Lee (2019)

Wysokinski (2019)

Subtotal ( 1 -squared $=0.0 \%, p=0.893$ )

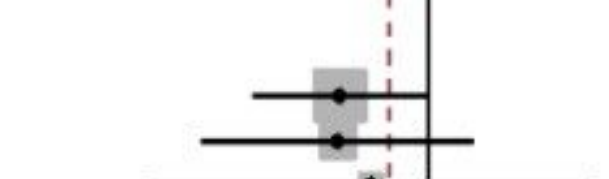

$0.42(0.18,0.99) \quad 5.35$

$0.41(0.11,1.54) \quad 2.47$

$0.57(0.07,4.83) \quad 0.82$

$0.46(0.02,11.92) \quad 0.37$

$0.71(0.53,0.95) \quad 32.30$

$0.65(0.30,1.42) \quad 5.12$

$0.63(0.17,2.42) \quad 1.69$

$0.98(0.25,3.77) \quad 1.32$

$2.00(0.41,9.91) \quad 0.75$

$0.93(0.32,2.67) \quad 2.23$

$0.69(0.54,0.87) \quad 52.42$

Apixaban

McBane II (2019)

Agnelli (2020)

Wysokinski (2019)

Subtotal ( $(1$-squared $=64.7 \%, p=0.059$ )

DOAC

Alzghari (2018)

Xiang (2018)

Pheips (2018)

Pritchard (2017)

Uppuluri (2017)

Subtotal ( 1 -squared $=0.0 \%, p=0.875$ )

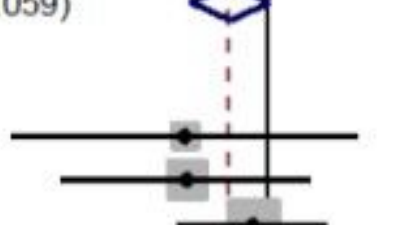

$0.10(0.01,0.82) \quad 2.79$

$0.68(0.43,1.09) \quad 13.40$

$1.37(0.58,3.22) \quad 2.71$

$0.69(0.47,1.03) \quad 18.90$

Overall (I-squared $=0.0 \%, p=0.886$ )

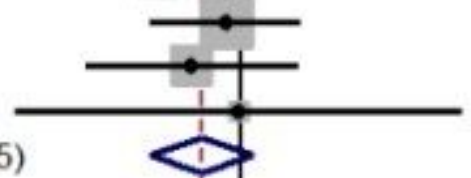

$0.44(0.08,2.40) \quad 1.18$

$0.45(0.13,1.54) \quad 2.52$

$0.86(0.41,1.80) \quad 4.82$

$0.62(0.22,1.76) \quad 2.88$

$0.98(0.11,8.63) \quad 0.51$

$0.68(0.41,1.12) \quad 11.90$

$0.69(0.58,0.81) \quad 100.00$

$\begin{array}{llll}1 & 1 & 1 \\ 0128 & 1 & 78\end{array}$

\section{Figure 3}

Subgroup analyses of ORs of different DOACs for VTE recurrence. 


Study
ID

\section{Figure 4}

Forest plot of ORs of DOACs compared with LMWHs for DVT recurrence in RCTs and retrospective studies, respectively. 
Study

ID

$\%$

OR $(95 \% \mathrm{Cl}) \quad$ Weight

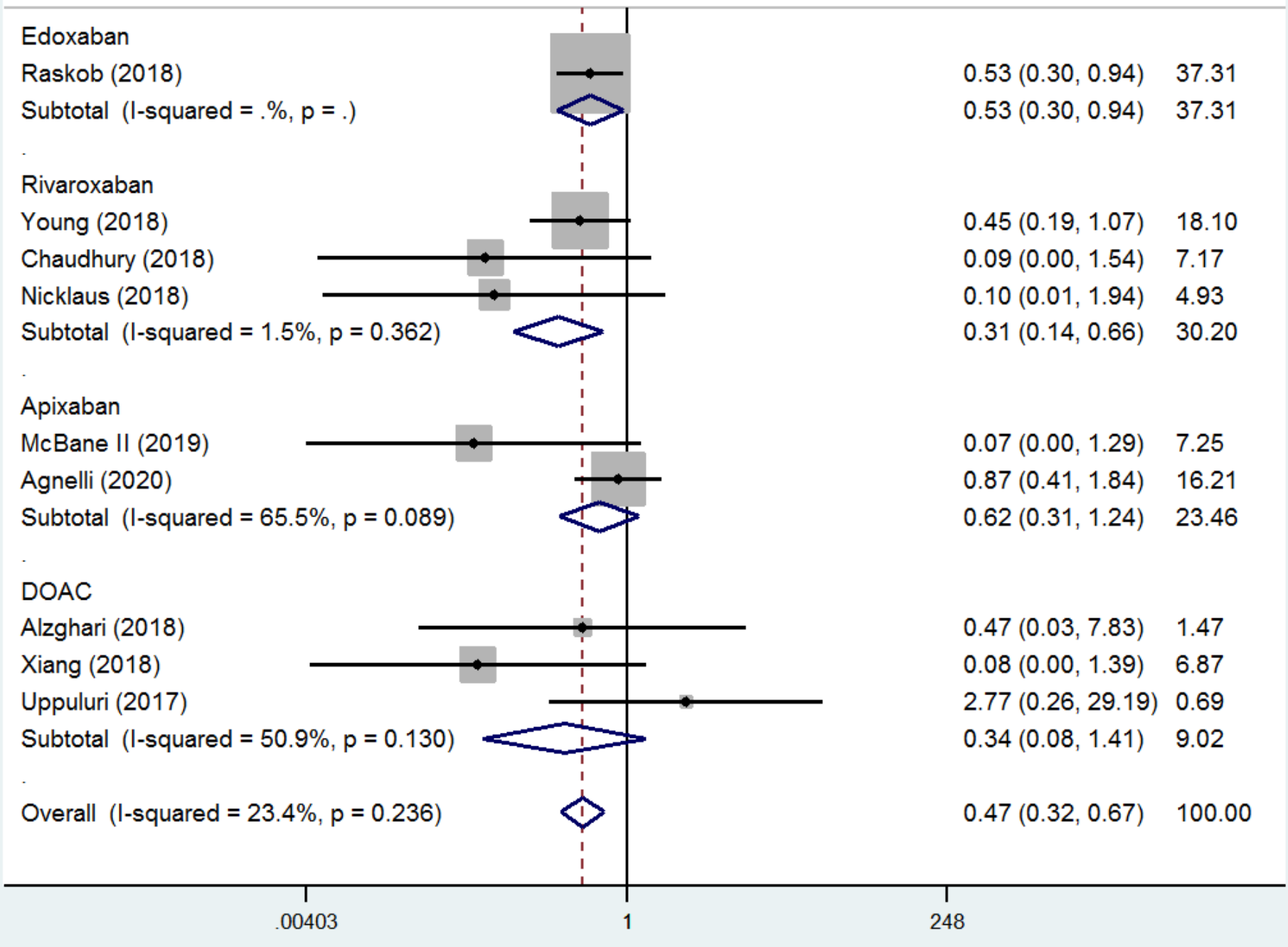

\section{Figure 5}

Subgroup analyses of ORs of different DOACs for DVT recurrence. 
Study

ID

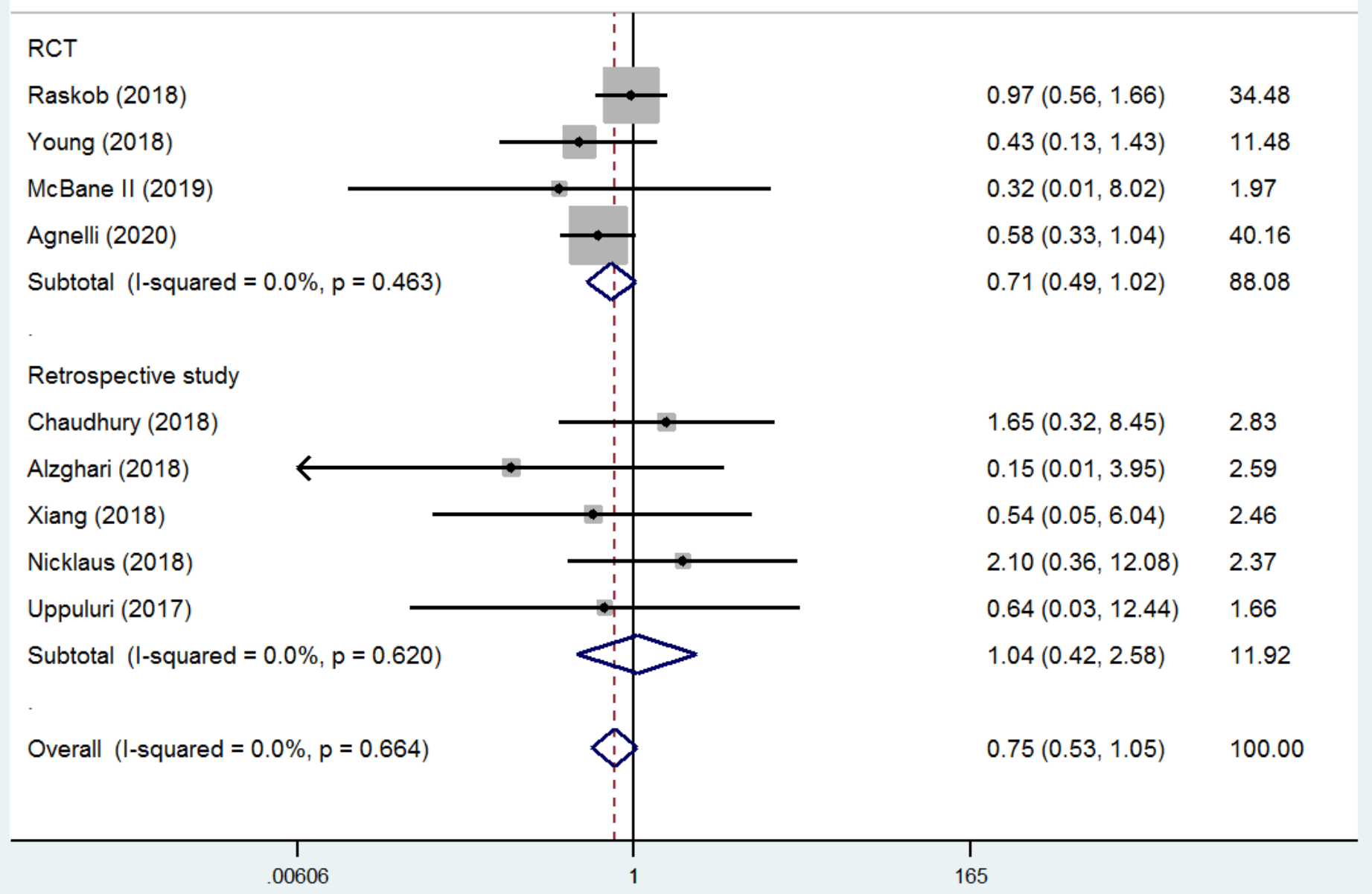

\section{Figure 6}

Forest plot of ORs of DOACs compared with LMWHs for PE recurrence in RCTs and retrospective studies, respectively. 
Study

ID
$\%$
OR $(95 \% \mathrm{Cl}) \quad$ Weight

$0.97(0.56,1.66) \quad 15.88$

$1.88(0.68,5.19) \quad 3.40$

$0.19(0.01,4.06) \quad 1.51$

$0.96(0.53,1.74) \quad 13.22$

$1.02(0.71,1.47) \quad 34.00$

$2.55(0.42,15.53) \quad 0.87$

$1.20(0.36,3.94) \quad 2.85$

$0.96(0.14,6.40) \quad 1.31$

$1.47(0.14,14.92) 0.76$

$1.68(1.04,2.72) \quad 15.68$

$0.71(0.12,4.41) \quad 1.68$

$1.01(0.44,2.30) \quad 6.74$

$0.33(0.12,0.89) \quad 10.16$

$0.87(0.39,1.97) \quad 7.45$

$0.64(0.03,12.44) \quad 0.77$

$0.56(0.18,1.70) \quad 5.68$

$0.93(0.48,1.83) \quad 10.61$

$2.31(0.48,11.17) \quad 1.44$

$1.05(0.81,1.36) \quad 66.00$

$1.04(0.84,1.28) \quad 100.00$

Overall $(\mathrm{I}$-squared $=0.0 \%, p=0.490)$

Lee (2019)

Subtotal $(\mathrm{I}$-squared $=6.4 \%, \mathrm{p}=0.382$ )

$$
.00919
$$

1

109

Figure 7

Forest plot of ORs of DOACs compared with LMWHs for major bleeding events in RCTs and retrospective studies, respectively. 
Study

ID

OR $(95 \% \mathrm{CI})$

$\%$

Edoxaban

Raskob (2018)

Subtotal $($ l-squared $=. \%, p=$.

Rivaroxaban

Young (2018)

Chaudhury (2018)

Ross (2016)

Signorelli (2019)

Streiff (2018)

Simmons (2018)

Lee (2019)

Lee (2019)

Wysokinski (2019)

Subtotal (I-squared $=0.0 \%, p=0.714$ )

Apixaban

McBane II (2019)

Agnelli (2020)

Wysokinski (2019)

Subtotal $($ I-squared $=0.0 \%, p=0.581$ )

DOAC

Alzghari (2018)

Xiang (2018)

Phelps (2018)

Pritchard (2017)

Uppuluri (2017)

Subtotal (I-squared $=0.0 \%, p=0.590$ )
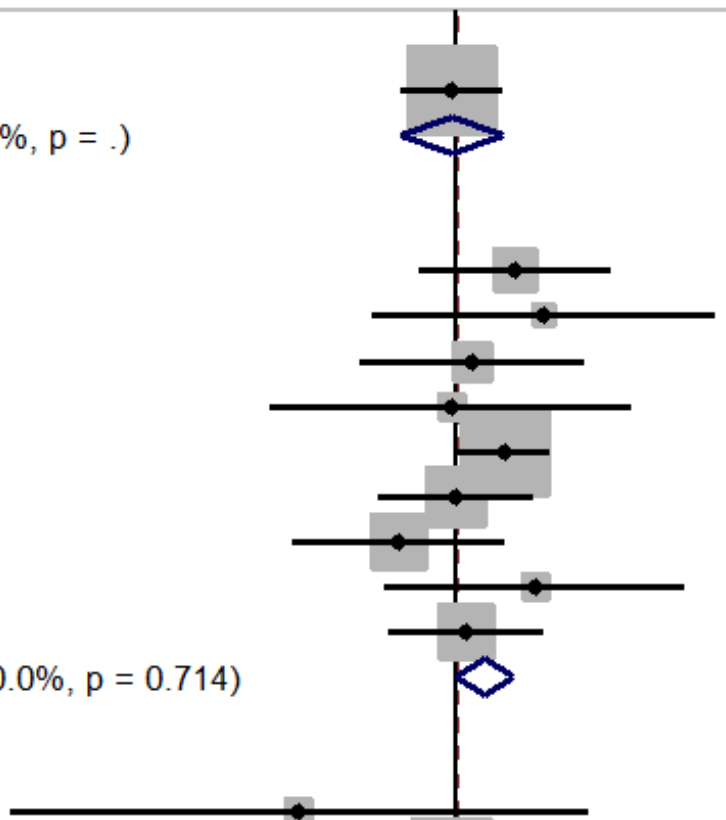

$0.97(0.56,1.66)$

15.32

$0.97(0.56,1.66)$

15.32

$1.88(0.68,5.19) \quad 3.28$

$2.55(0.42,15.53) \quad 0.84$

$1.20(0.36,3.94) \quad 2.75$

$0.96(0.14,6.40) \quad 1.26$

$1.68(1.04,2.72) \quad 15.14$

$1.01(0.44,2.30) \quad 6.50$

$0.56(0.18,1.70) \quad 5.48$

$2.31(0.48,11.17) \quad 1.39$

$1.12(0.49,2.55) \quad 6.09$

$1.36(1.01,1.82) \quad 42.75$

$0.19(0.01,4.06) \quad 1.46$

$0.96(0.53,1.74) \quad 12.76$

$0.80(0.35,1.82) \quad 7.62$

$0.85(0.53,1.37) \quad 21.84$

Overall (I-squared $=0.0 \%, p=0.538$ )

$1.47(0.14,14.92) \quad 0.73$

$0.71(0.12,4.41) \quad 1.62$

$0.33(0.12,0.89) \quad 9.81$

$0.87(0.39,1.97) \quad 7.19$

$0.64(0.03,12.44) \quad 0.74$

$0.61(0.35,1.05) \quad 20.09$

$1.04(0.84,1.28) \quad 100.00$

\section{Figure 8}

Subgroup analyses of ORs of different DOACs for major bleeding events. 
Study

ID
OR $(95 \% \mathrm{Cl})$

$\%$

Weight

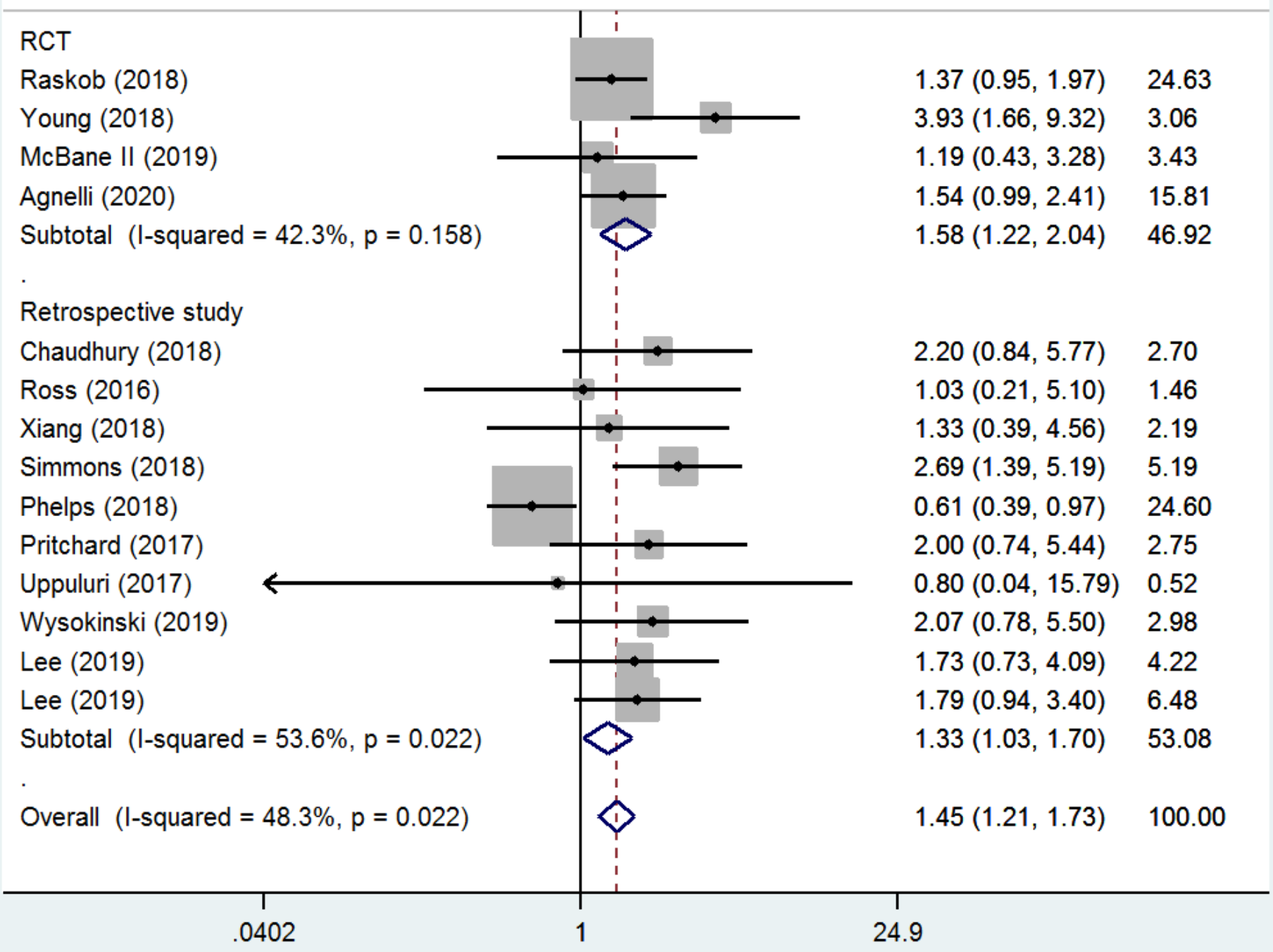

\section{Figure 9}

Forest plot of ORs of DOACs compared with LMWHs for CRNMBs in RCTs and retrospective studies, respectively. 
Study

ID

OR $(95 \% \mathrm{Cl})$

$\%$

Weight

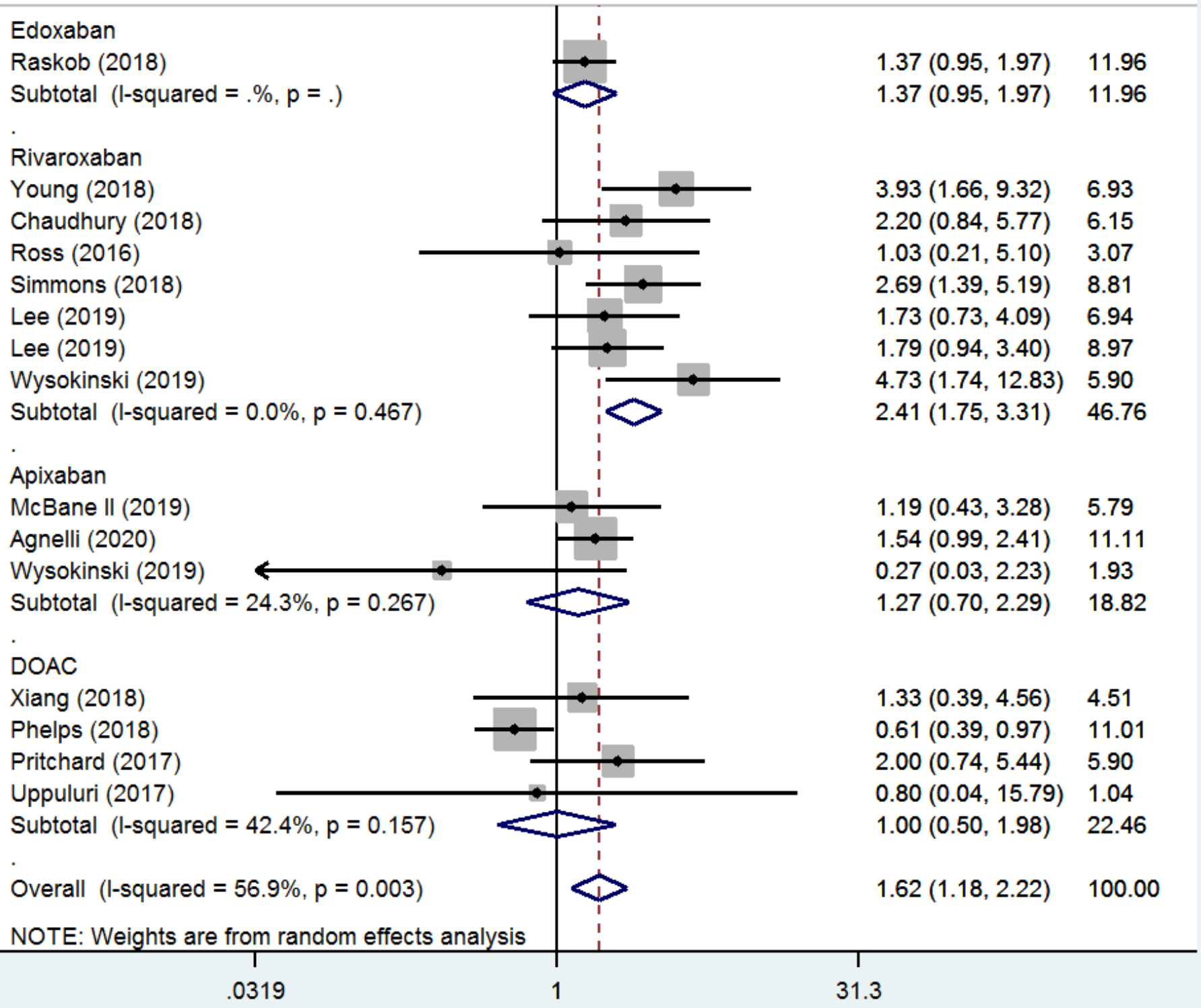

Figure 10

Subgroup analyses of ORs of different DOACs for CRNMBs.

\section{Supplementary Files}

This is a list of supplementary files associated with this preprint. Click to download.

- SupplementaryFig.1.tif

- SupplementaryFig.2.tif 
- SupplementaryFig.3.tif

- SupplementaryFig.4.tif

- SupplementaryFig.5.tif

- SupplementaryFig.6.tif

- SupplementaryFig.7.tif

- Supplementarytable1.doc

- Supplementarytable2.doc 